\title{
Balancing Long Lifetime and Satisfying Fairness in WBAN Using a Constrained Markov Decision Process
}

\author{
Yingqi Yin, ${ }^{1}$ Fengye Hu, ${ }^{1}$ Ling Cen, ${ }^{2}$ Yu Du, ${ }^{1}$ and Lu Wang ${ }^{1}$ \\ ${ }^{1}$ College of Communication Engineering, Jilin University, Changchun 130022, China \\ ${ }^{2}$ Institute for Infocomm Research, Singapore 138632 \\ Correspondence should be addressed to Fengye Hu; hufy@jlu.edu.cn
}

Received 26 June 2014; Revised 9 September 2014; Accepted 10 September 2014

Academic Editor: Lingyang Song

Copyright (c) 2015 Yingqi Yin et al. This is an open access article distributed under the Creative Commons Attribution License, which permits unrestricted use, distribution, and reproduction in any medium, provided the original work is properly cited.

\begin{abstract}
As an important part of the Internet of Things (IOT) and the special case of device-to-device (D2D) communication, wireless body area network (WBAN) gradually becomes the focus of attention. Since WBAN is a body-centered network, the energy of sensor nodes is strictly restrained since they are supplied by battery with limited power. In each data collection, only one sensor node is scheduled to transmit its measurements directly to the access point (AP) through the fading channel. We formulate the problem of dynamically choosing which sensor should communicate with the AP to maximize network lifetime under the constraint of fairness as a constrained markov decision process (CMDP). The optimal lifetime and optimal policy are obtained by Bellman equation in dynamic programming. The proposed algorithm defines the limiting performance in WBAN lifetime under different degrees of fairness constraints. Due to the defect of large implementation overhead in acquiring global channel state information (CSI), we put forward a distributed scheduling algorithm that adopts local CSI, which saves the network overhead and simplifies the algorithm. It was demonstrated via simulation that this scheduling algorithm can allocate time slot reasonably under different channel conditions to balance the performances of network lifetime and fairness.
\end{abstract}

\section{Introduction}

With the increasing development of wireless communication technology and wireless sensor network (WSN), the emerging wireless body area network (WBAN) provides great opportunities in real-time healthcare monitoring, fitness, entertainment, and consumer electronics applications without restricting the activities of users [1]. WBAN is a dynamic network with sensor nodes in, on, or around the body for continuous monitoring of physiological parameters with capabilities of real-time processing and data communication as well. Device-to-device (D2D) communication is a hot technology, which allows direct communication between closely located devices using the licensed band [2]. Networks can benefit from improved reliability, robustness, and coverage provided by $\mathrm{D} 2 \mathrm{D}$ communications [3]. However, there are some challenges in $\mathrm{D} 2 \mathrm{D}$ communication. Power efficiency is one of the difficulties due to the fact that proximate devices must use very low transmission power for reliable communication [4]. Besides, source allocation is another research hotspot in
D2D communication. In [5], a fair resource allocation problem for D2D communications was studied in orthogonal frequency division multiple access- (OFDMA-) based wireless cellular networks. In [6], a genetic algorithm (GA) with frequency hopping technique was proposed to optimally select the number of frequency channels required in the system and then allocate these frequency channels to the UE clusters for D2D communication. The sensor nodes in the dynamic network of WBAN and the AP can be considered as proximate devices in $\mathrm{D} 2 \mathrm{D}$ communication. Using the processing methods for references is thus a good choice to improve the performance of WBAN.

In the literature, some methods have been proposed aiming at improving the performance of WBAN in the aspects of MAC (media access control) protocol design [7], data fusion [8], security [9], and so forth. However, WBAN is still in its early development stage, and there are some challenges we must conquer before it can be widely applied [10]. For instance, limited battery energy makes a huge demand of lifetime. Lifetime extension in WBAN has attracted 
increasing interest of researchers. Transmission scheduling algorithms have been explored for maximizing the lifetime of WSN in some publications, for example, [11, 12]. In [13], a general formula of lifetime in WSN was proposed, which demonstrated that channel state information (CSI) and residual energy information (REI) were major parameters in the issue of lifetime maximization. CSI is also an essential factor in closed-loop wireless communication systems [14]. A dynamic transmission scheduling scheme dubbed dynamic protocol for lifetime maximization (DPLM) was proposed in [15], which has been demonstrated to be asymptotically optimal in network lifetime. As shown in [16], the problem of lifetime maximization was formulated as a stochastic shortest path Markov decision process. An iterative algorithm was developed to find a Pareto-optimal solution for maximizing the lifetime of WSN in [17].

These transmission scheduling schemes care about only lifetime maximization in WSN under the condition of homogeneous traffic requirements. However, this cannot work well when sensor nodes require different data transmission rates. In WBAN, different types of physiological parameters such as body temperature, blood pressure, electrocardiograph (ECG), and electroencephalograph (EEG) are monitored, which requires various data rates for sensor nodes. The transmission scheduling schemes mentioned above can cause socalled unfairness in selection where some channel conditions cannot be satisfied during a long time and consequently some sensor nodes will not be selected.

The concept of fairness has been intensively investigated for resource allocation in wireless network. As is shown in [15], a pure opportunistic transmission scheduling scheme was proposed to carry out transmission in the best channel condition. This scheme is throughput optimal; in the meantime, it can cause unfair resource allocation. Several algorithms have been presented to improve the system performance under fairness constraints. The authors in [18] take network throughput and fairness of user equipment into account by performing interference management. An opportunistic fair scheduling scheme for CDMA (code division multiple access) networks was developed in [19], which relates the average transmission of users to their fair weights achieved. In [20], an optimization framework was proposed to balance the performance of lifetime and fairness. To allocate time slots based on the demands of sensor nodes, a utility-based allocation method was adopted in [21]. In [22], a fair resource allocation approach was proposed for D2D communication in wireless cellular networks.

Due to limited battery energy in WBAN, increasing network lifetime and meantime maintaining fairness are conflicting with each other within limits. Balancing network lifetime and fairness performance is important in WBAN. In the literature, however, very few research efforts have been made to address the issue of balance between lifetime and fairness. To the best of our knowledge, there is no widely accepted unified framework that can be effectively used for accurate evaluation of performance under different tradeoff between lifetime and fairness. To address this problem, a novel centralized transmission scheduling scheme that utilizes a constrained Markov decision process (CMDP) and

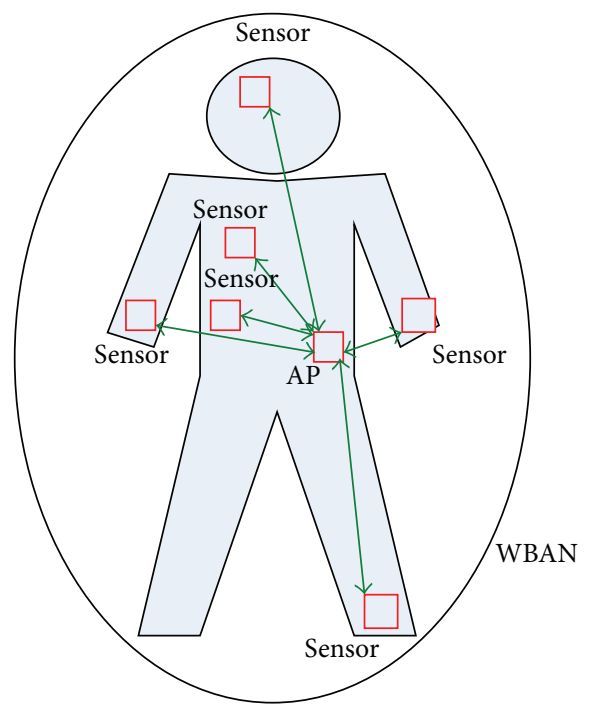

Figure 1: WBAN model.

a distributed one are proposed in this paper, which can both extend lifetime and maintain fairness of sensor nodes, aiming at balancing lifetime and fairness in WBAN.

The remainder of this paper is organized as follows. In Section 2, the model of WBAN and the formulation of lifetime are described. In Section 3, an optimization framework using CMDP is presented. The proposed fair weights transmission scheduling scheme is presented in Section 4. Finally, we conclude the paper in Section 5.

\section{WBAN Model}

2.1. WBAN Model. We consider a WBAN which consists of an access point (AP) and $N$ sensors with initial energy $E_{\text {in }}$. We adopt a star topology. Each sensor transmits its own equalsized data packets that are directly transmitted to the AP through a common channel as shown in Figure 1. Assume a block channel that remains constant within each transmission slot and varies independently in between different slots. During communication in WBAN, the strength of signals can be fading due to reflection, diffraction, energy absorption, shadowing by body, and body posture. A theoretical channel model may trace back to the fundamental theories of electromagnetic propagation and require precise modeling of a specific situation, which is too complex and exceeds our research.

Each sensor node measures a certain physiological parameter and transmits corresponding data packets directly to the AP through the fading channel. The received signal can be expressed as

$$
y_{i}(t)=h_{i} \cdot x_{i}(t)+n(t)
$$

where $x_{i}(t)$ is the transmitted signal, $h_{i}$ is the channel fading in sensor $i$, and $n(t)$ is additive white Gaussian noise with power spectrum density $N_{0} / 2$ that is the identical in all 
sensors. Assume that a block-fading channel of $T$ seconds with channel gain expressed as

$$
C_{i}=\left|h_{i}\right|^{2}
$$

keeps constant within each time slot. Here $C_{i}$ is exponentially distributed in the condition of independent Rayleigh fading. The AP broadcasts beacon signals to initiate the data collection process and each sensor node estimates their channel condition. Since the AP is usually a mobile phone or another personal digital assistant (PDA) containing enough energy, the energy consumption of the AP is not considered in this work. We suppose that sensors can ensure satisfying transmission and reduce unnecessary energy consumption by adjusting their transmission power according to channel conditions. In practical applications, sensors can only transmit at a finite number of power levels according to hardware limitations [23]. Let $M$ be the number of power levels and $a_{1}, a_{2}, \ldots, a_{m}, \ldots$, and $a_{M}$ denote the power scaling factors of a transmitter, where $0 \leqslant a_{1}<\cdots<a_{M} \leqslant 1$. The power level is then restricted to a finite set shown as

$$
P_{i}=\left\{a_{k} P_{\max }\right\}_{k=1}^{M},
$$

where $P_{i}$ is the transmission power available for sensor $i$ transmitting a data packet to the AP if it is scheduled and $P_{\max }$ is the maximum transmission power that transmitter can achieve. For simplicity, based on the Shannon theorem, the transmission rate of sensor $i$, denoted by $v_{i}$, can be expressed as

$$
\begin{aligned}
v_{i} & =B \log _{2}\left(1+\frac{C_{i} P}{N_{0} B}\right) \leq B \log _{2}\left(1+\frac{C_{i} P_{i}}{N_{0} B}\right) \\
& =B \log _{2}\left(1+\frac{C_{i} a_{k} P_{\max }}{N_{0} B}\right),
\end{aligned}
$$

where $p$ is the desired value of transmission power in theory and $B$ is the bandwidth. In (4), $a_{k}$ adopts the minimum value for the sake of matching with the inequality. Since $a_{k}$ depends on the current channel gain and the transmission rate related to the sensor $i$, the energy consumption for data transmitting of sensor $i$ in data collection can be written as

$$
E_{\mathrm{txi}}=a_{k} P_{\max } \cdot T+E_{c}
$$

where $E_{c}$ is the energy consumption of the transmitter circuit and it is identical for all sensor nodes.

2.2. Formulation of Lifetime in WBAN. A general formula of lifetime in WSN is described in [13]. We adopt this lifetime concept in WBAN, which expresses WBAN lifetime as

$$
E\{L\}=\frac{N \cdot E_{\text {in }}-E\left\{E_{w}\right\}}{N \cdot e+E\left\{E_{\mathrm{tx}}\right\}} .
$$

In (6), $N_{\text {in }}$ is the initial energy of sensor nodes, $E_{\text {tx }}$ is the expected transmission energy consumed in one round of data collection, $E_{w}$ is the expected wasted energy, and $e$ is the energy required by a sensor for CSI acquisition. The wasted energy is set to be the total unused energy when the lifetime completes. It can be expressed as

$$
E_{w}=\sum_{i=1}^{N} E_{w i}
$$

where $E_{w i}$ is the wasted energy of sensor $i$. A sensor node is supposed to be dead when its residual energy is lower than the transmitter circuit consumption; that is, under any channel condition it has no enough energy to transmit. A WBAN is considered to be dead when any sensor node in this network is dead. In this paper, we express the lifetime of a WBAN as the number of data allocations before the network dies.

\section{Optimal Transmission Scheduling}

In each time slot, only one sensor node is scheduled to transmit its measurements directly to the AP through the fading channel. We assume that the instantaneous CSI of all sensors is available to the AP. In this section, we formulate the problem of dynamically choosing which sensor should communicate with the AP to maximize network lifetime under the constraint of fairness as a CMDP. We propose a centralized transmission scheduling algorithm that maximizes network lifetime under different constraint of fairness. The optimal lifetime and optimal policy are achieved by Bellman equation in dynamic programming. The optimal policy using global CSI defines the limiting performance in network lifetime for the model specified in Section 2.

3.1. Fairness Index. Fairness is in general a critical factor in performance studies. Particularly in distributed networks where resources are shared by a number of users, fair allocation is extremely important and fairness is considered as an important criterion in the design of a WBAN.

In the MAC layer of IEEE 802.15.6 specification, time is divided into superframes, each with equal length. The superframes consist of four periods: control period, contention access period (CAP), contention-free period (CFP), and inactive period. The CFP is further divided into a number of time slots. We focus on the time division multiple access(TDMA-) based protocol, in which data packets are mainly transmitted in the CFP. Therefore, this is a time-slotted network, where time is the resource to be allocated among the sensor nodes.

In the literature, Jain's fairness index [24] has been widely used as a measure of network-wise fairness performance. Let $T_{i}$ denote the actual transmission time of sensor $i$ and let $O$ denote the total transmission times. $b_{i}$ indicates the weighting factor, which expresses the degree of importance of sensor $i$. Then the normalized time allocation of sensor $i$ can be given as

$$
x_{i}=\frac{T_{i}}{b_{i} O} .
$$


If $x_{i}$ is used to represent the allocation received by sensor $i$ in a network with $N$ competitive sensors, the fairness index for the network we proposed in this work can be expressed as

$$
f=\frac{\left(\sum x_{n}\right)^{2}}{N \sum x_{n}^{2}} .
$$

A transmission scheduling scheme is considered to be perfectly fair if $f=1$. A higher value of $f$ indicates higher fairness level among the sensor nodes and the converse is true.

3.2. CMDP Formulation. We use an infinite horizon CMDP to model the sensor selection problem aiming at achieving different performance tradeoff between lifetime and fairness. The major components are elaborated below.

3.2.1. State Space. Let e be residual energy and let $\mathbf{w}$ and $f$ be the transmission energy requirement and fairness index in each time slot, respectively. The network state space characterized by $\mathbf{e}, \mathbf{w}$, and $f$ can be expressed as

$$
\mathbf{S}=\{i=(\mathbf{e}, \mathbf{w}, f)\}
$$

When the network lifetime expires, it reaches a special termination state $S_{t}$ expressed as

$$
S_{t}=\left\{(\mathbf{e}, \mathbf{w}, f): \forall\left\{e_{n}: e_{n}<\varepsilon_{1}\right\} \text { or } \mathbf{e}<\mathbf{w}\right\} \text {, }
$$

where $e_{n}<E_{c}$ indicates that the residual energy of the $n$th sensor reduces below the transmitter circuit consumption and $\mathbf{e}<\mathbf{w}$ indicates a transmission failure.

3.2.2. Action Space. The set of actions is denoted by $\mathbf{A}$. The action space in state $i=(\mathbf{e}, \mathbf{w}, f) \in \mathbf{S}$ can be described as follows:

$$
A(i)=A[(\mathbf{e}, \mathbf{w}, f)]=\left\{n: e_{n} \geq w_{n}\right\}
$$

The set of actions consists of the indexes of all sensors that support the current transmission.

3.2.3. Transition Probability. Assume that sensor $n$ will be selected for transmitting after action $a$ is applied. If the state at time $t$ is $i$ and action $a$ is taken, then the probability that the next state is $j$ can be calculated as

$$
P_{i a j}=p\left(w^{\prime}\right) 1_{\left[e^{\prime}=e-\mathbf{I}_{n} w_{n}\right]},
$$

where $p\left(w^{\prime}\right)=\operatorname{Pr}\left\{W=w^{\prime}\right\}$ is the probability mass function of $W$ determined by channel fading for a predefined set $W$ of transmission energy. Let $\mathbf{I}_{n}=(0, \ldots, 0,1,0, \ldots, 0)$ be a $1 \times N$ unit vector that the $n$th element is set to be $1.1_{[x]}$ denote the indicator function that $1_{[x]}=1$ if $x$ is true and zero otherwise.

3.2.4. Transmission Reward. After each of the transmissions, the network is assigned a unit reward until the network enters a terminating state. In other words, if the network is in state $i=(\mathbf{e}, \mathbf{w}, f)$, the instantaneous reward $R(i)$ in this time slot is defined as

$$
R(i)=1_{\left[i \in S \backslash S_{t}\right]}
$$

when state $i \in S$ and $i \notin S_{t}$. The network lifetime, denoted by $L$, can be described as the accumulated total reward until the network enters a terminating state in $S_{t}$.

3.2.5. The Constraint. In state $i$, an action is considered as a feasible action if the fairness index $f$ of the network is larger than a given threshold $f_{n}$ after action $a$ is applied. This can be expressed as

$$
f(i, a) \leq f_{n}
$$

where $f(i, a)$ represents the fairness index of the next state in state $i$ after action $a$ is applied. The set of available actions in state $i$ is denoted by $A_{a}(i)$.

3.2.6. CMDP Formulation. Now we formulate the sensor scheduling problem in the form of CMDP. A transmission scheduling protocol is a policy $u$ in CMDP. A policy $u$ in the policy space $\mathbf{U}$ is a sequence; that is, $u=\left\{u_{0}, u_{1}, \ldots\right\}$, where $u_{t}: S \rightarrow\{1, \ldots, N\}$ specifies the sensor selected in the $t$ th time slot and $u_{t}\left(\cdot \mid i_{1}, a_{1}, i_{2}, a_{2}, \ldots, i_{t-1}, a_{t-1}, i_{t}\right)$ is a conditional probability measure over $\mathbf{A}$. Let $L_{u}(i)$ specify the expected network lifetime (the total reward in the CMDP) starting from state $i$ with policy $u$. The maximum expected lifetime $L^{*}(i)$ starting form state $i$ is given by

$$
L^{*}(i)=\max _{u} L_{u}(i)
$$

A policy $u^{*}$ is considered optimal if it obtains the maximum expected lifetime before the network reaches the terminating state; that is,

$$
L_{u^{*}}(i)=L^{*}(i), \quad \forall i \in S \backslash S_{t} .
$$

We define $U_{a}=\left\{u \in U: f_{u} \geqslant f_{n}\right\}$ as an available policy set. If $u^{*} \in U_{a}$ satisfies the condition $L\left(u^{*}\right) \geqslant L(u),\left(u \in U_{a}\right)$, $u^{*}$ is called the constrained optimal policy.

Hence, the constrained optimization problem is to find a feasible $u \in U$ that maximizes network lifetime $L$. The optimal sensor scheduling protocol is given by the constrained optimal policy $u^{*}$ in the above CMDP problem.

3.3. Optimal Policy. The maximum expected lifetime $L^{*}(i)$ starting form state $i$ with the fairness constraint can be obtained as the unique solution to the Bellman optimality equation shown as

$$
\begin{aligned}
& L^{*}(i)=L^{*}[(\mathbf{e}, \mathbf{w}, f)] \\
&=R(i)+\max _{n \in A(i)}\left\{\sum_{j \in S} p_{i a j} L^{*}(j)\right\}, \quad \forall i \in S, \\
& \text { s.t. } f_{j} \geq f_{n},
\end{aligned}
$$


where $f_{j}$ is the fairness index of the network in state $j$ and $f_{n}$ is the fairness threshold specified according to different application scenario.

Equation (18) can be rewritten as

$$
L^{*}(i)=L^{*}[(\mathbf{e}, \mathbf{w}, f)]=R(i)+\max _{n \in A_{a}(i)}\left\{\sum_{j \in \mathbf{S}} p_{i a j} L^{*}(j)\right\},
$$$$
\forall i \in S \text {. }
$$

An optimal policy $u$ for transmission scheduling protocol is given by

$$
u(i)=\underset{n \in A_{a}(i)}{\arg \max }\left\{\sum_{j \in S} p_{i a j} L^{*}(j)\right\}, \quad \forall i \in S \backslash S_{t} .
$$

Similar to [11], an equivalent modified Bellman's optimality equation can be expressed as

$$
L[\mathbf{e}, f]=\sum_{w} p(w)\left\{R[(\mathbf{e}, \mathbf{w})]+\max _{n \in A_{a}} L\left[\mathbf{e}-\mathbf{I}_{n} w_{n}\right]\right\} .
$$

Hence, the constrained optimal policy can also be expressed as

$$
u[(\mathbf{e}, \mathbf{w}, f)]=\arg \max _{n \in A_{a}} L\left\{\mathbf{e}-\mathbf{I}_{n} w_{n}\right] .
$$

3.4. Implementation and Overhead. We need to acquire network energy profile $\mathbf{e}$ and transmission energy requirement $\mathbf{w}$ to implement constrained Markov decision process using global CSI. We also need to understand how to realize the instantaneous channel for all sensors. The way of implementation is elaborated below. First, AP broadcasts a beacon signal to activate each sensor in the network at the start of a data collection slot. Each sensor then responds to the AP by sending pilot signals and acquiring global CSI. The AP estimates the channel station of all response sensors and realizes the transmission energy requirement $\mathbf{w}$ by the response signals from sensors. Next, according to the CMDP and the current network state $(\mathbf{e}, \mathbf{w}, f)$, the AP determines which sensor to be scheduled. Lastly, the AP broadcasts the ID of the selected sensor and the required transmission power level. Then, the chosen sensor on the required transmission power level reports its observed value to the AP. The AP can trace the network energy profile easily by knowing the scheduled sensor's ID and channel realizations of all sensors.

The main disadvantage of the CMDP is the huge energy consumption due to the fact that each sensor needs to consume energy to transmit pilot signals to acquire global CSI. Nevertheless, any sensor scheduling protocols would sacrifice the network lifetime.

3.5. Numerical Results. In this section, the transmission scheduling using CMDP is evaluated in simulation. The performance is evaluated by using lifetime and fairness index.

Firstly, we compare the lifetime of the optimal transmission scheduling with the following four scheduling protocols:

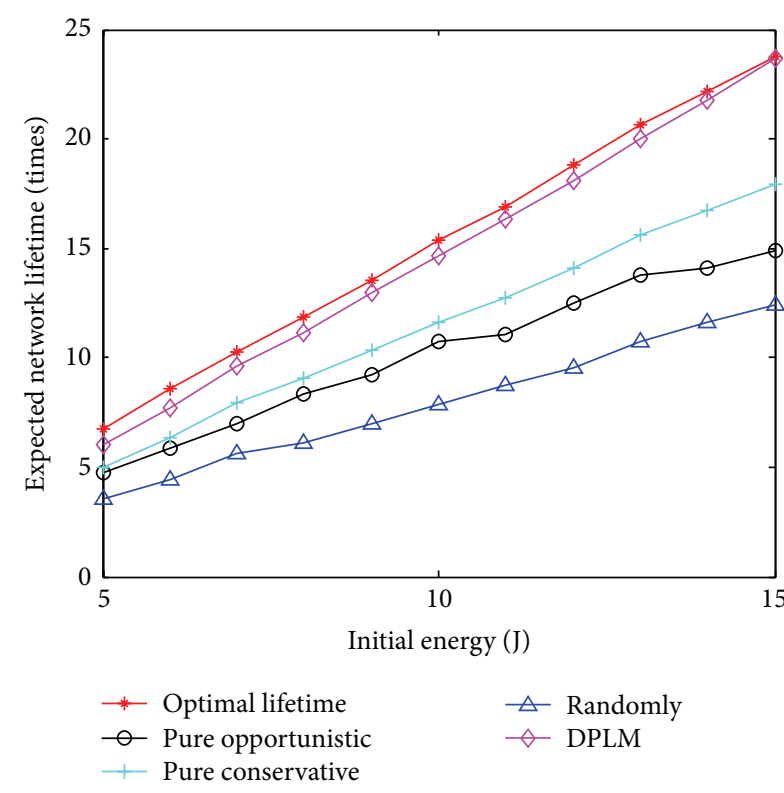

FIGURE 2: Expected lifetime for optimal lifetime, pure opportunistic, pure conservative, randomly, and DPLM schemes.

(1) the AP randomly scheme which selects a sensor to transmit; (2) the pure opportunistic scheme which selects the sensor with the best channel condition; (3) the pure conservative approach which chooses sensor with the most residual energy; (4) the DPLM protocol which selects the sensor with the largest ratio between the residual energy and the current transmission consumption. As shown in Figure 2, when the WBAN adopts optimal transmission scheduling, pure opportunistic, pure conservative, randomly, and DPLM schemes, respectively, the network lifetime is proportional to the initial energy of sensor nodes. Obviously, the randomly scheme performs the worst. The pure conservative approach outperforms the pure opportunist approach. Among these, the optimal transmission scheduling achieves the perfect performance.

In WBAN, every sensor node monitors various physiological parameters with different degrees of importance. That is to say, the sensor nodes have different weighting factors in the network. Then, we illustrate the performance of the optimal transmission scheduling scheme under the condition of equal weighting factors and different weighting factors.

3.5.1. Equal Weighting Factors. In the simulation of Figure 3, we set that the weighting factors of every sensor are the equal value of $1 / 3$. The fairness thresholds are assigned to be 0.3 , $0.5,0.7$, and 0.9 , respectively, which represent the fairness requirement in various application scenarios. As shown in Figure 3, the network lifetime improves with the increase of initial energy of sensor nodes. The higher the requirement of fairness, the lower the network lifetime.

3.5.2. Different Weighting Factors. In order to compare with the above simulation, the weighting factors are assigned to be $0.7,0.2$, and 0.1 , respectively. The set of fairness thresholds 


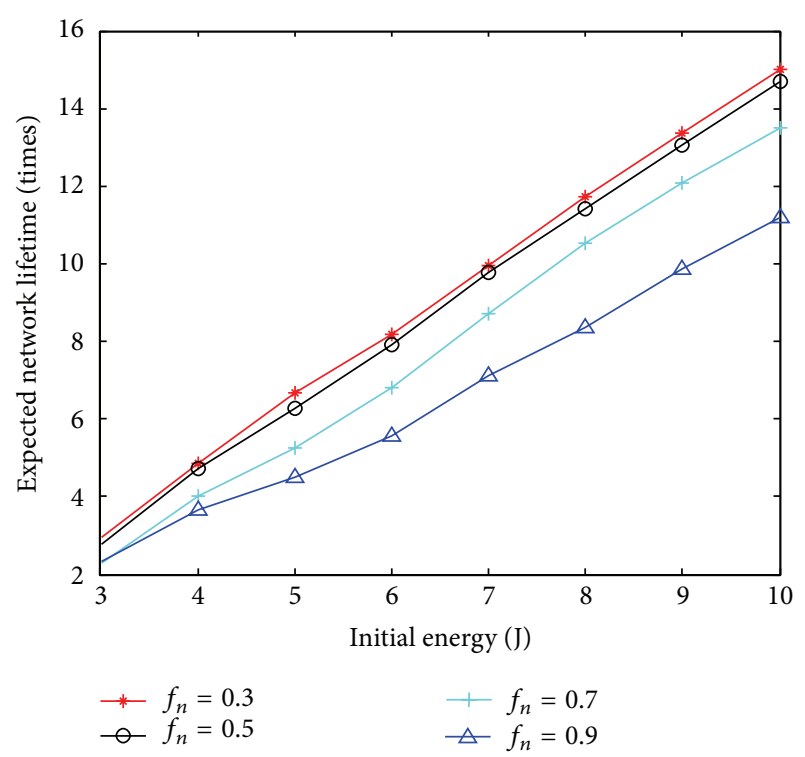

Figure 3: Expected lifetime for optimal lifetime with equal weighting factors.

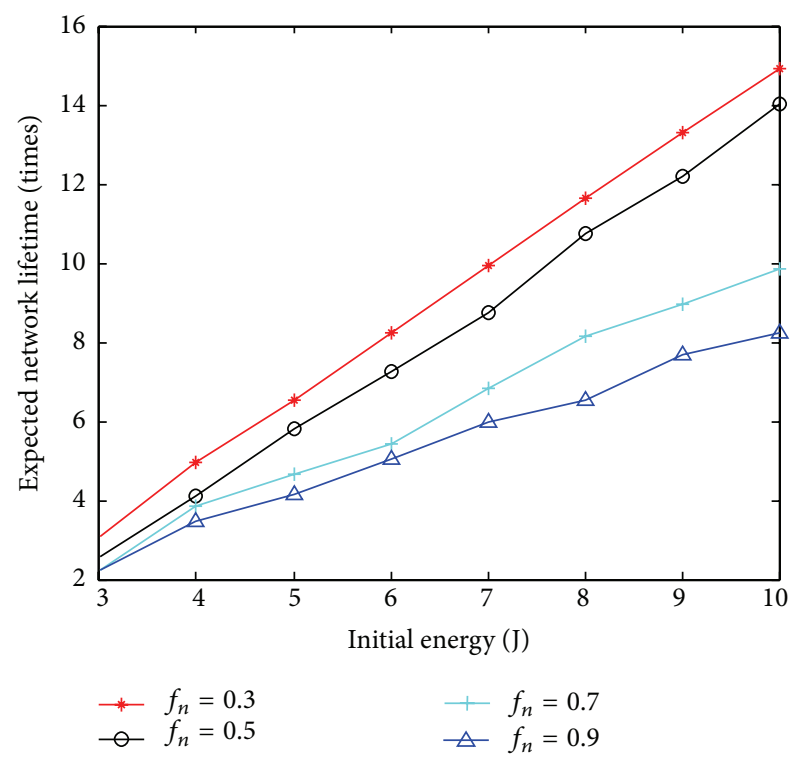

FIGURE 4: Expected lifetime for optimal lifetime with different weighting factors.

are equal to Figure 3. As shown in Figure 4, lifetime also follows the change of fairness, when all sensors have identical initial energy, the lower the requirements for fairness, and the longer the lifetime. The basic difference between Figure 3 and Figure 4 is that the layouts of curves in Figure 4 are more diffuse. This means that the constraint of fairness has a greater impact on the performance of network lifetime under the condition of different weighting factors. As shown in (8) and (9), the weighting factors work as a part of fairness indexes. When the weighting factors of different sensors are not identical and especially when the weighting factors of some sensors are much larger than the others, the proposed algorithm tends to select the sensor with a higher weighting factor rather than the one with a longer lifetime, in order to ensure fairness performance. Therefore, if fairness is highly required in WBAN, the weighting factors have an intensive impact on network lifetime; otherwise, the weighting factors have a smaller impact on lifetime.

By comparing Figures 3 and 4, it can be observed that the proposed optimal transmission scheduling scheme can achieve different degrees of tradeoff between network lifetime and fairness by utilizing different degrees of fairness constraint.

\section{Proposed Fair Weights Scheduling Scheme}

Due to the defect of large implementation overhead in acquiring global CSI, we put forward a novel distributed scheduling algorithm that adopts local CSI, which saves the network overhead and simplifies the algorithm.

4.1. Design Principle. In this section, a novel distributed transmission scheduling scheme, which is named as fair weights scheme in this paper, is proposed. Fair weights consist of functions of CSI, REI, exponentially weighted moving average (EWMA) of data rate, and expected data rate. The proposed fair weights scheme satisfies the following design principles:

(i) maximize lifetime of the whole network through weights of both CSI and REI;

(ii) maintain fairness. To do so, the transmission scheme should adjust fair weights grounded on old samples of sensor nodes' transmission condition.

4.2. Fair Weights Scheme. DPLM has been proved to be asymptotically optimal in lifetime, in which we based on in lifetime maximization. At the beginning of data allocation, the sensor whose current energy consumption demands the smallest portion of its residual energy for transmission is selected based on the DPLM scheme. Accordingly, the energy-efficiency index is defined as a ratio of residual energy and expected energy consumption is expressed as

$$
e_{i}=\frac{E_{i}}{E_{\mathrm{tx} i}}
$$

where $E_{i}$ is the residual energy of sensor $i$.

In order to maintain fairness, we adopt the EWMA of data rate. The scheme holds a running average $\overline{v_{i}}$ of data rate, which is achieved by using exponentially weighted moving average of each newly obtained sample during decision history. In our scheme, at the beginning of each scheduling interval $t$, the exponentially weighted moving average of data rate for sensor $i$ is updated as

$$
\overline{v_{i}(n)}=\partial_{i} v_{i}(n-1)+\left(1-\partial_{i}\right) \overline{v_{i}(n-1)},
$$

where $v_{i}(n-1)$ is the transmission rate of sensor $i$ at scheduling interval $n-1$. If sensor $i$ is not scheduled to transmit at scheduling interval $n-1, v_{i}(n-1)$ is assigned to be 
the value of 0. In (10), $\partial_{i} \in[1,0]$ is a constant, which determines the rate of exponential decay of the previous samples. A larger $\partial_{i}$ results in rapid decay and the converse is true. Considering this, we can tune $\partial_{i}$ according to the physiological parameter to improve fairness. For example, ECG monitoring requires higher data rate than body temperature monitoring. Therefore, a higher $\partial_{i}$ will be set for ECG monitoring sensor to make $\overline{v_{i}}$ decrease radically when it fails to be scheduled for several times. On the contrary, lower $\partial_{i}$ should be set for body temperature monitoring sensors requiring lower data rate. It can be seen that this scheme is desirable in the sense that it attempts to compensate for unfairness of recent allocations as much as possible.

Letting $R_{i}$ be the ratio of $\overline{v_{i}}$ to the expected data rate of sensor $i$, denoted by $R_{0 i}$, then it can be expressed as

$$
R_{i}=\frac{\overline{v_{i}}}{R_{0 i}} .
$$

In (25), $R_{i}$ represents the deviation between $\overline{v_{i}}$ and $R_{0 i}$. Note that $R_{0 i}$ is different for different sensors processing different kinds of physiological parameters. Then the fair weight of sensor $i$ is defined as

$$
W_{i}=\frac{e_{i}}{R_{i}}=\frac{e_{i}}{\overline{v_{i}} / R_{0 i}} .
$$

The fair weights in (26) are used to determine which sensor node will transmit its measurements to the AP through the common channel during each round of data collection by exploiting CSI, REI, data rate requirement, and decision history. If sensor $i$ has not been scheduled for a long time, the value of $\overline{v_{i}}$ decreases severely. As a result, $R_{i}$ decreases and the fair weight $W_{i}$ increases to achieve larger possibility for seizing the channel. The fair weights are calculated periodically to accommodate the channel condition. The objective of the proposed scheme is to improve fairness performance without sacrificing excessive network lifetime, which can overcome the shortage of the existing distributed transmission scheduling schemes. Consequently, it is able to achieve a desired balance between increasing lifetime and maintaining fairness in the design of a WBAN.

4.3. Distributed Implementation. In this part, we consider the implementation of the proposed distributed transmission scheduling scheme. Here, we adopt opportunistic carrier sensing [10] in our implementation. The basic idea is to match the fair weight of each sensor node with the backoff function of carrier sensing. It provides a distributed solution in the searching of global maximum. At the beginning of each scheduling interval, the AP broadcasts a beacon and each sensor node estimates its channel information and calculates the predefined fair weight. After that, each sensor node maps its fair weight $w$ to a backoff time using a predetermined backoff function $f(w)$ and then listens to the channel. If $f(w)$ is designed to be a strictly decreasing function of $w$ as shown in Figure 5, this opportunistic carrier sensing will ensure that only the sensor with the maximum fair weight will transmit data. The propagation delay among sensors is assumed to be

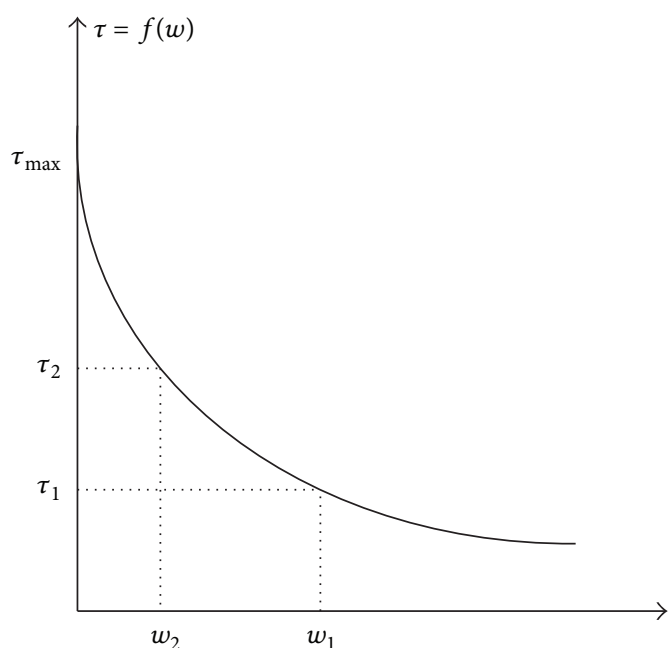

FIGURE 5: Opportunistic carrier sensing.

negligible, and the sensor will be scheduled to transmit if its backoff time $\tau_{i}$ expires before the other sensors transmit, which indicates that the sensor with the maximum fair weight will seize the channel. In the case that multiple nodes have identical values of fair weight $W$, collision will happen. This will be considered in our future work.

4.4. Fairness Criterion. A fairness factor is defined for fairness evaluation in this section, which is expressed as

$$
F=\sum_{x=1}^{N} \sum_{y=1}^{N}\left|g_{x}-g_{y}\right|
$$

where the $g_{x}$ and $g_{y}$ denote the ratio between actual transmission times and the given expected transmission times of sensors $x$ and $y$, respectively, in a fixed time period.

4.5. Numerical Results. In this section, simulation results are provided to illustrate the effectiveness of the proposed fair weights scheme. Lifetime and fairness factor are used as performance metrics. The proposed fair weights scheduling scheme is compared with the pure opportunistic scheme that uses only CSI and the DPLM scheme that utilizes the energyefficiency index to select the sensor transmitting data packets to the AP. Without loss of generality, the estimation of channel conditions that is identical in all schemes and the energy consumption of carrier sensing are not considered here.

A WBAN consisting of 5 sensor nodes is considered. The power spectrum density of noise is set to be $-70 \mathrm{Dbm} / \mathrm{Hz}$, and the bandwidth is $5 \times 10^{6} \mathrm{~Hz}$. Assume that the channel gain follows an exponential distribution and is set to be 1 . The energy consumption of transmitter circuitry, denoted by $E_{c}$, is set to be 0.001 Joule. The transmission rate $v_{i}$ is $250 \mathrm{k}$ bits per second for all sensors. The expected data rates are gradually increased; that is, $R_{0 i}=\{0.5,1,2,5,10\}$ kbps for sensors $1-$ 5. It means that the index of a sensor node is proportion to data packets that are to be transmitted. In our scheme, the parameter $\partial_{i}$ is initially set to be $\{0.5,0.6,0.7,0.8,0.9\}$, 


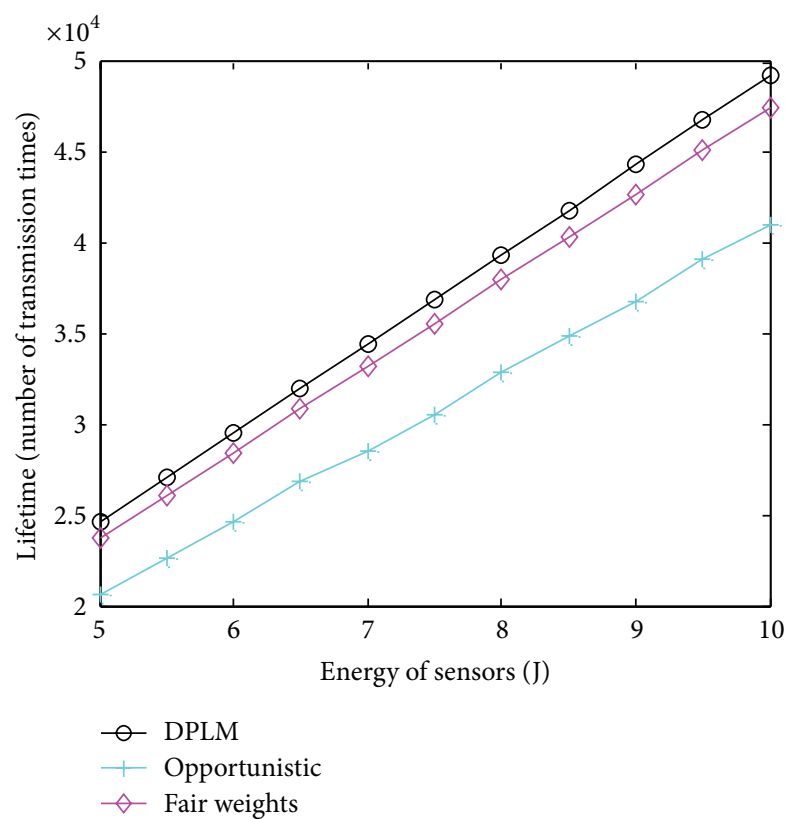

FIGURE 6: Lifetime versus energy of sensors achieved from pure opportunistic, DPLM, and fair weights scheme.

for sensors $1-5$, respectively, and will be adjusted according to miscellaneous physiological parameters.

Figure 6 shows the lifetime of the WBAN designed using different methods, that is, pure opportunistic, the DPLM, and the fair weights schemes. The network lifetime improves with the increase of initial energy of sensor nodes. It can be seen from the figure that the pure opportunistic scheme ignoring REI achieves the worst performance. DPLM has the best performance of lifetime without considering fairness constraint. Although considering the condition of fairness constraint, the WBAN from the fair weights scheme has the lifetime equal to DPLM. The small gap between the performance of DPLM and the fair weights scheme is due to the fact that the fairness constraint will force the scheme to select the sensor that has not been scheduled for a long time regardless of their CSI and REI.

Figure 7 shows the fairness factor versus 300 of scheduling intervals achieved by using the pure opportunistic, DPLM, and fair weights schemes. As the number of scheduling intervals increases, the fairness factor tends towards being stable. The scheduling scheme satisfies the rate requirements of sensor nodes on a large time scale while the fair weights periodically computed compensate unfairness. It can be found from Figures 6 and 7 that, by sacrificing a small amount of lifetime within an acceptable level, the fair weights scheme is able to greatly enhance the fairness performance. In the meantime, the network designed using the fair weights scheduling scheme achieves a balance between lifetime and fairness performance.

In order to illustrate the fairness performance more clearly, the scheduled time slots of each sensor node versus time are compared when their traffic is scheduled by DPLM and fair weights schemes, which are shown in Figure 8.

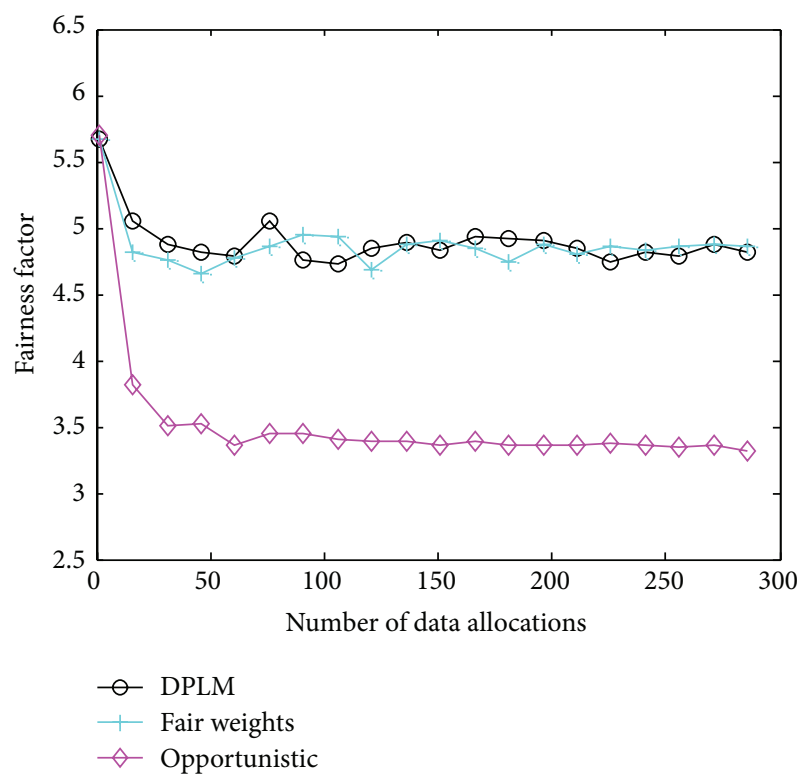

FIGURE 7: Fairness factor achieved from pure opportunistic, DPLM, and fair weights scheme.

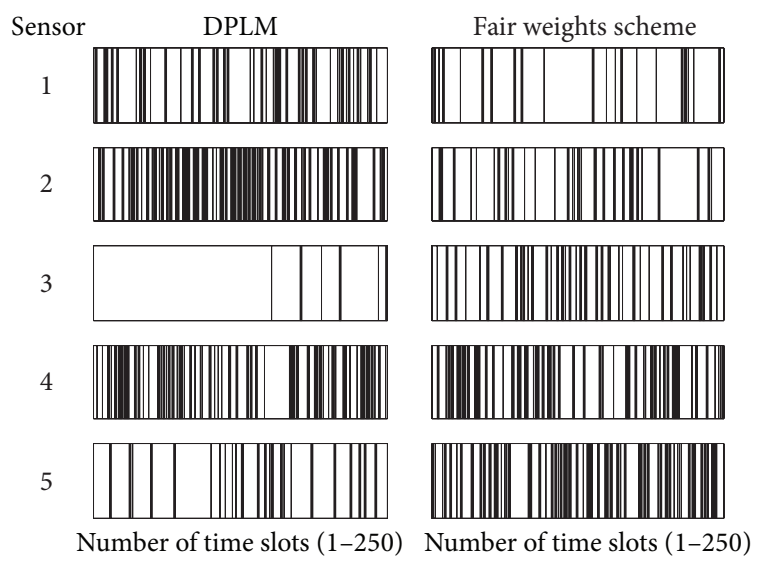

FIGURE 8: Scheduled time slots of sensors 1 to 5 versus time for DPLM and fair weights scheme.

The range of time slots in simulation is from 1 to 250 . In order to make the contrast more obvious, we set that the channel gains of sensor 3 and sensor 5 are persistently severe. The simulation illustrated in Figure 8 shows that the DPLM scheme ignores sensor node with low energy-efficiency index, such as sensor 3 and sensor 5 . This result leads to severe unfairness in data rate allocation. As shown in the corresponding simulation of fair weights scheme, the sensors that are ignored by DPLM get compensations and the sensors with higher expected data rate obtain more priorities to transmit their data packets to the AP.

It can be seen from the simulation results that the proposed fair weights scheme can effectively allocate time slots to balance the performance of lifetime and fairness in various channel conditions. 


\section{Conclusions}

Lifetime and fairness are two primary concerns in WBAN. In this paper, an infinite horizon CMDP is proposed to model the sensor selection, which is able to achieve different degrees of performance tradeoff between lifetime and fairness. The simulation results show that the proposed scheduling algorithm can obtain optimal lifetime utilizing optimal policy under different degrees of constraints. To overcome the defect of large application overhead and complexity of the abovementioned algorithm, a distributed transmission scheduling scheme using local CSI is also proposed to achieve a balance between lifetime and fairness. Taking heterogeneous rate requirements into consideration, energy-efficiency index and exponentially weighted moving average of data rate are employed, which are devoted to lifetime and fairness, respectively. It can be demonstrated from simulation results that the proposed scheduling scheme is able to achieve much better fairness performance at only a very little cost of lifetime in the tradeoff between lifetime and fairness.

\section{Conflict of Interests}

The authors declare that there is no conflict of interests regarding the publication of this paper.

\section{Acknowledgment}

This work is supported by the National Natural Science Foundation of China (nos. 61074165 and 61273064).

\section{References}

[1] M. A. Hanson, H. C. Powell Jr., A. T. Barth et al., "Body area sensor networks: challenges and opportunities," Computer, vol. 42 , no. 1, pp. 58-65, 2009.

[2] 3GPP, "Feasibility study for proximity services (prose)," Tech. Rep. TR 22.803 V0.2.0, 3GPP, 2012.

[3] A. H. Tsai, C. L. Wang, H. J. Huang et al., "Intelligent resource management for device-to-device (D2D) communications in heterogeneous networks," in Proceedings of the 15th International Symposium on Wireless Personal Multimedia Communications (WPMC '12), pp. 75-79, 2012.

[4] L. B. Le, "Fair resource allocation for device-to-device communications in wireless cellular networks," in Proceedings of the IEEE Global Communications Conference (GLOBECOM '12), pp. 5451-5456, Anaheim, Calif, USA, December 2012.

[5] Y.-H. Lee, H.-W. Tseng, C.-Y. Lo et al., "Using genetic algorithm with frequency hopping in device to device communication (D2DC) interference mitigation," in Proceedings of the International Symposium on Intelligent Signal Processing and Communications Systems (ISPACS '12), pp. 201-206, New Taipei, Taiwan, November 2012.

[6] H. Zhang, Y. Fu, and J. Liu, "Optimal transmission distance of mean progress and mean transport in device-to-device networks," in Proceedings of the International Conference on Cyberspace Technology (CCT '13), pp. 332-337, IET, Beijing, China, November 2013.
[7] A. Boulis, D. Smith, D. Miniutti, L. Libman, and Y. Tselishchev, "Challenges in body area networks for healthcare: the MAC," IEEE Communications Magazine, vol. 50, no. 5, pp. 100-116, 2012.

[8] F. Hu, S. Li, T. Xue, and G. Li, "Design and analysis of low-power body area networks based on biomedical signals," International Journal of Electronics, vol. 99, no. 6, pp. 811-822, 2012.

[9] D. Halperin, T. Kohno, T. S. Heydt-Benjamin, K. Fu, and W. H. Maisel, "Security and privacy for implantable medical devices," IEEE Pervasive Computing, vol. 7, no. 1, pp. 30-39, 2008.

[10] M. Patel and J. Wang, "Applications, challenges, and prospective in emerging body area networking technologies," IEEE Wireless Communications, vol. 17, no. 1, pp. 80-88, 2010.

[11] R. Madan, S. Cui, S. Lall, and A. Goldsmith, "Cross-layer design for lifetime maximization in interference-limited wireless sensor networks," in Proceedings of the 24th Annual Joint Conference of the IEEE Computer and Communications Societies (INFOCOM '05), vol. 3, pp. 1964-1975, March 2005.

[12] K. Cohen and A. Leshem, "A time-varying opportunistic approach to lifetime maximization of wireless sensor networks," IEEE Transactions on Signal Processing, vol. 58, no. 10, pp. 53075319, 2010.

[13] Y. Chen and Q. Zhao, "On the lifetime of wireless sensor networks," IEEE Communications Letters, vol. 9, no. 11, pp. 976-978, 2005.

[14] L. Song, Z. Han, Z. Zhang, and B. Jiao, "Non-cooperative feedback-rate control game for channel state information in wireless networks," IEEE Journal on Selected Areas in Communications, vol. 30, no. 1, pp. 188-197, 2012.

[15] Y. Chen and Q. Zhao, "An integrated approach to energy-aware medium access for wireless sensor networks," IEEE Transactions on Signal Processing, vol. 55, no. 7, part 1, pp. 3429-3444, 2007.

[16] Y. Chen, Q. Zhao, V. Krishnamurthy, and D. Djonin, “Transmission scheduling for optimizing sensor network lifetime: a stochastic shortest path approach," IEEE Transactions on Signal Processing, vol. 55, no. 5, pp. 2294-2309, 2007.

[17] J. C. Dagher, M. W. Marcellin, and M. A. Neifeld, "A theory for maximizing the lifetime of sensor networks," IEEE Transactions on Communications, vol. 55, no. 2, pp. 323-332, 2007.

[18] F. Wang, L. Song, Z. Han, Q. Zhao, and X. Wang, "Joint scheduling and resource allocation for device-to-device underlay communication," in Proceedings of the IEEE Wireless Communications and Networking Conference (WCNC '13), pp. 134-139, IEEE, April 2013.

[19] M. Mehrjoo, M. K. Awad, M. Dianati, and X. S. Shen, "Design of fair weights for heterogeneous traffic scheduling in multichannel wireless networks," IEEE Transactions on Communications, vol. 58, no. 10, pp. 2892-2902, 2010.

[20] H. T. Cheng and W. Zhuang, "An optimization framework for balancing throughput and fairness in wireless networks with QoS support," IEEE Transactions on Wireless Communications, vol. 7, no. 2, pp. 584-593, 2008.

[21] M. Dianati, X. Shen, and K. Naik, "Cooperative fair scheduling for the downlink of CDMA cellular networks," IEEE Transactions on Vehicular Technology, vol. 56, no. 4 I, pp. 1749-1760, 2007.

[22] L. B. Le, "Fair resource allocation for device-to-device communications in wireless cellular networks," in Proceedings of the IEEE Global Communications Conference (GLOBECOM '12), pp. 5451-5456, December 2012. 
[23] S. Xiao, A. Dhamdhere, V. Sivaraman, and A. Burdett, "Transmission power control in body area sensor networks for healthcare monitoring," IEEE Journal on Selected Areas in Communications, vol. 27, no. 1, pp. 37-48, 2009.

[24] R. Jain, D. M. Chiu, and W. R. Hawe, A Quantitative Measure of Fairness and Discrimination for Resource Allocation in Shared Computer System, Eastern Research Laboratory, Digital Equipment Corporation, 1984. 

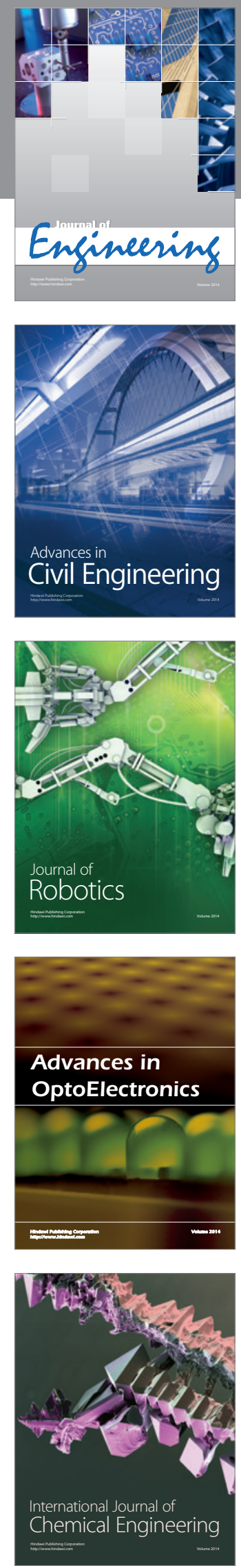

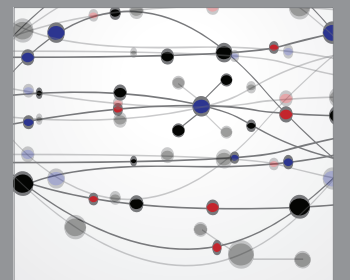

The Scientific World Journal
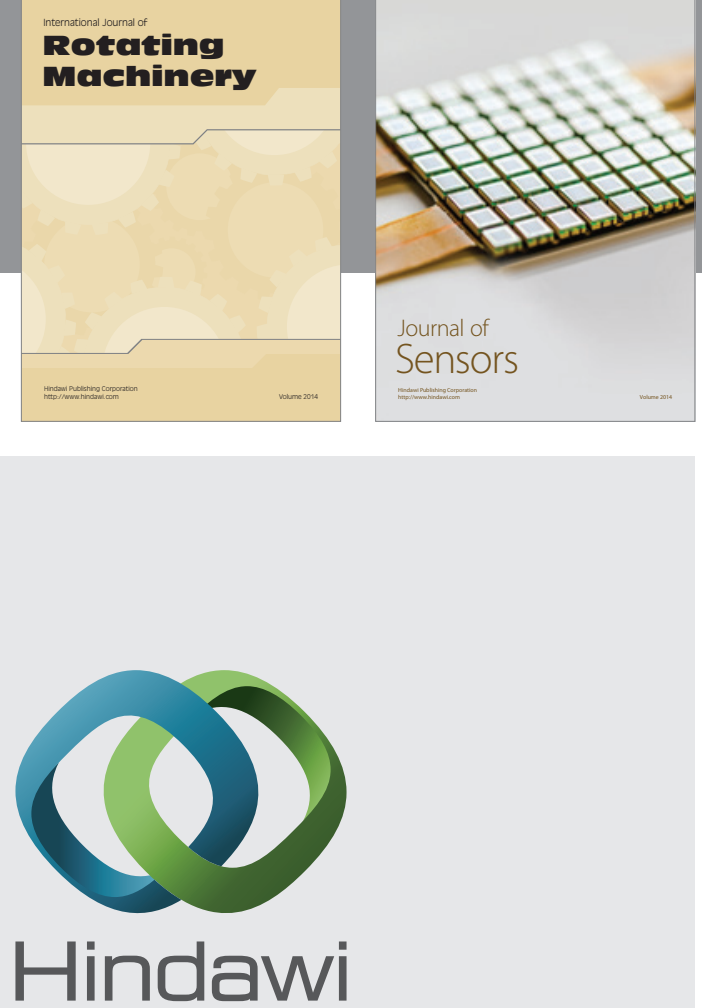

Submit your manuscripts at http://www.hindawi.com
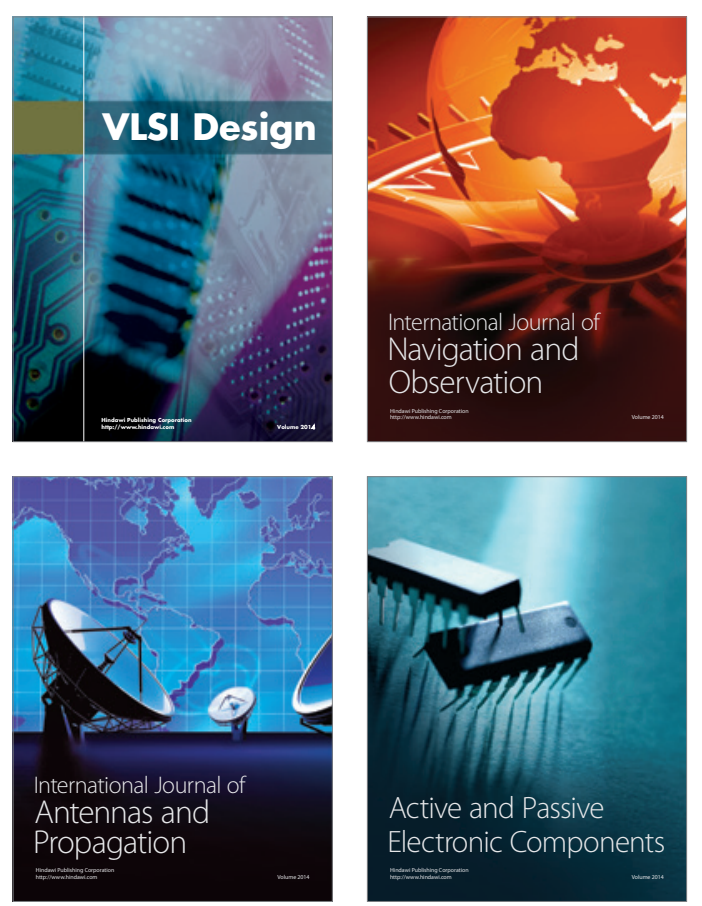
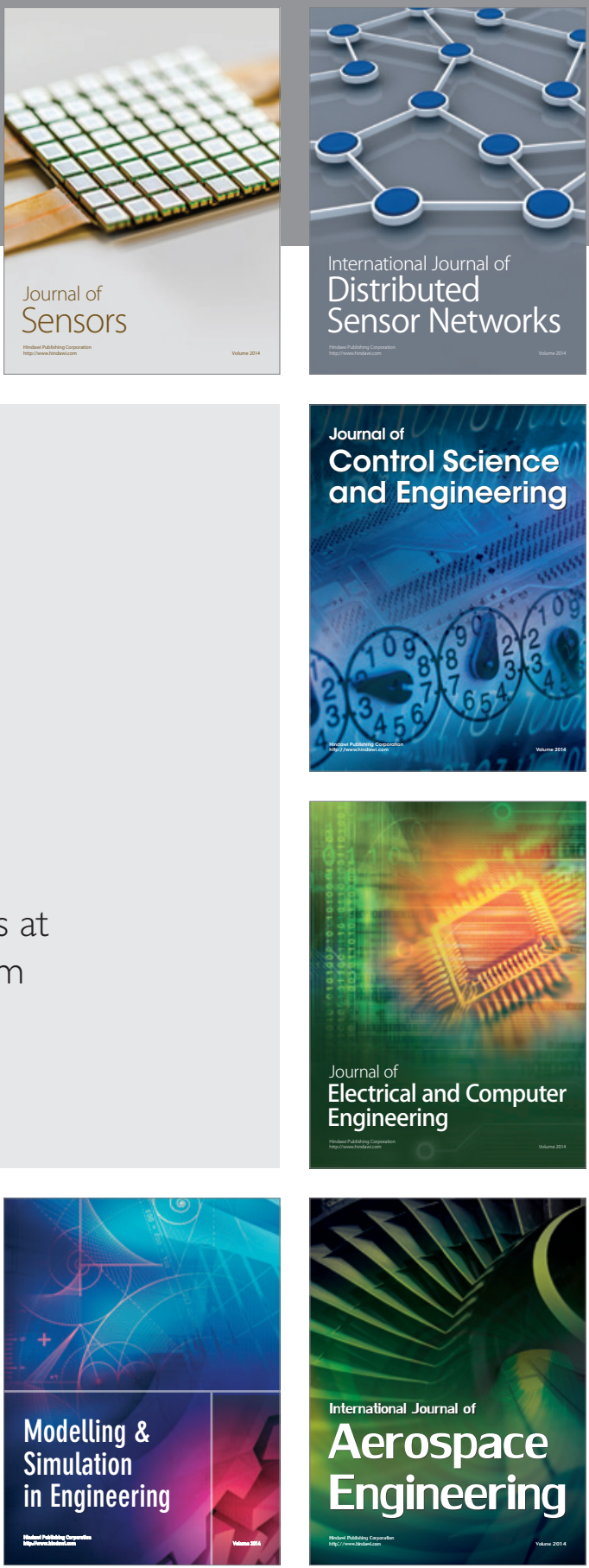

Journal of

Control Science

and Engineering
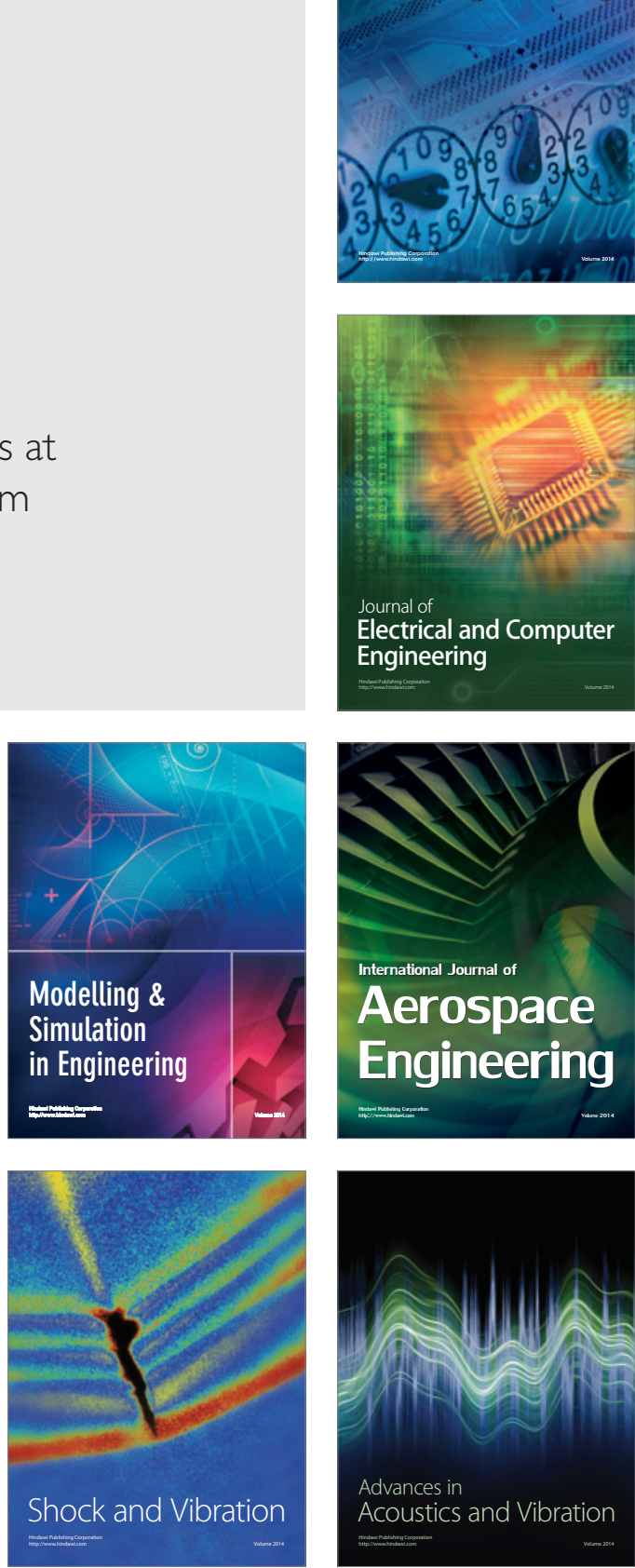